\title{
Human Recognition using Single-Input-Single-Output Channel Model and Support Vector Machines
}

\author{
Sameer Ahmad Bhat ${ }^{1}$, Abolfazl Mehbodniya ${ }^{2}$, Ahmed Elsayed Alwakeel ${ }^{3}$, Julian Webber ${ }^{4}$ and Khalid Al-Begain ${ }^{5}$ \\ Dept. of Electronics and Communications Engineering, Kuwait College of Science and Technology (KCST), Kuwait ${ }^{1,2,3,5}$. \\ Graduate School of Engineering Science, Osaka University, Japan ${ }^{4}$.
}

\begin{abstract}
WiFi based human motion recognition systems mainly rely on the availability of Channel State Information (CSI). Embedded within WiFi devices, the present radio subsystems can output CSI that describes the response of a wireless communication channel. Radio subsystems as such, use complex hardware architectures that consume lots of energy during data transmission, as well as exhibit phase drift in the sub-carriers. Although human motion recognition (HMR) based on multicarrier transmission systems show better classification accuracy, transmission of multiple sub-carriers results in an increase in the overall energy consumption at the transmitter. Apparently CSI based systems can be perceived as process intensive and power hungry devices. To alleviate the process intensive computing and reduce energy consumption in $\mathrm{WiFi}$, this study proposes a human recognition system that uses only one radio carrier frequency. The study uses two software defined radios and a machine learning classifier to identify four humans, and the study results show that human identification is possible with $99 \%$ accuracy using only one radio carrier. The results of this study will have an impact on the development process of smart sensing systems, particularly those that relate to healthcare, authentication, and passive monitoring and sensing.
\end{abstract}

Keywords-Motion detection; pattern recognition; received signal strength indicator; Software Defined Radio (SDR); supervised learning

\section{INTRODUCTION}

In recent years, the role of smart environments has attracted most of the research communities across the globe, and the research activities undertaken by such communities, are transforming the existing natural, or made-man setups to smart environments. The areas that are influenced by these transformations also include indoor sensing, pattern recognition and classification systems, and smart environments. Smart home applications, spanning across various domains, enable support to build smart home environments, and human motion sensing environments, in particular, enable support to motion sensing, analysis, and evaluation of ambient environmental settings, or parameters, as a response to human activities.

At present, various state-of-the-art analytical methods have been devised to explore the analogous, and discriminative physiological and behavioral characteristics of humans, so as to model human motion behavior as well as to recognize different human motion patterns. A smart home is realized as a subunit in a smart environment, wherein human motion recognition, and localization applications may be deployed, both in indoor and outdoor environments. Indeed device free passive indoor localization [1] has been of great interest to researchers, and it plays a key role in the applications that enable assisted living facilities for elderly, children, physically challenged, and in smart home, etc. [2], [3], [4].

The combined motion sensing approaches and their reasoning deliver context-aware data from human motion, as well as from the analysis of human activities. The data collected is then next employed to provide personalized support in many applications [5]. Human motion sensing, analysis and prediction is classified into three categories: vision-based systems, wearable sensor-based systems, and RF based systems [6][7]. Sensing systems based on the approach of vision sensing are classified under the category of passive sensing systems. Sensing systems as such, use cameras as a light sensor for tracking human motion patterns [8] [9]. Human behavioral patterns captured in images can be processed using the computer vision and machine learning techniques. Typically, images captured with a camera, often needs a camera to have sufficient ambient light, and insufficient lighting effects visibility, which leads to significant decrease, or even no sensing capabilities in cameras. Moreover, any physical barriers such as walls completely alienate camera based sensing systems.

Wearable sensor-based approach [10] [11] [12], is one of the alternatives to monitoring human activities. Wearable sensing needs attaching sensors to the human body, and often pose challenges of electrical wiring, power supply management and in particular mainly cause inconvenience. Consequently, elderly patients abstain to carry electrical wires and monitoring sensors. Typically, subjects' data are recorded with inertial sensors such as gyroscopes, accelerometers, or magnetometers, enabling human motion data acquisition as electrical signals varying over time [13]. For consistent observation and monitoring of subjects' necessitates subjects carrying monitoring devices, often demanding power supply and other accessories items to supply. Thus, proposing solutions to eliminate sensor deployment on the human body is imperative and directs research to incorporate passive sensing and monitoring elements.

The shadowing effects left over by any moving targets intercepting line of sight (LoS) path between the transmitter and receiver, enable tracking of objects in motion in indoor environments [14] [15] [16]. An interception caused by a human walking across the LOS of the RF signal, results in variations in the received strength signal (RSS) at the receiver [17]. To Identify and track the unique human motion patterns out of RSS, requires analyzing the embedded unique human motion signatures, using various methods of signal processing.

On the other hand, advancements in wireless technology are driving researchers to devise solutions exploiting wireless communication systems in localization and pattern recogni- 
tion based applications. In fact, several attempts addressing issues concerning motion detection [18][19], gesture detection [20][21], and facial recognition systems [22] have been successful. With current wireless devices embedding multiple radio sub-units, allow sub-carriers for data communications. However, SDRs can also provide estimated Channel State Information (CSI), and many commercially available off-theshelf (COTS) devices support CSI data directly via in-built subsystems.

Although CSI based localization and pattern recognition studies reveal real higher performances, developed systems still exhibit challenges such as increased processing complexity, portability, adaptability, unreliability, lower precision, and inefficient system designs. Solutions based on existing wireless communication systems infrastructure, no doubt extend the scope of research in the current context, however, processing multiple sub-carriers in CSI based systems is of concern. Statistical CSI data retrieved either from commercially or customized firmware modified routers provides human activity and gesture data [23]. Recent attempts made using CSI of WiFi devices have shown higher motion recognition accuracies, nonetheless, it solely relies on available WiFi channels for monitoring. Therefore, developing efficient system designs will significantly impact potential applications implementing elementary and straightforward prototyping methods of localization and pattern recognition. This study aims to address the problem of human recognition. Our proposed solution bases on one sub-carrier frequency only, rather than multiple subcarrier frequencies, to identify and classify human motion patterns using machine learning.

The study will impact future works that relate to human recognition systems, employing SISO channel model of communication instead of using CSI based systems. The proposed testbed in this study can be used for further investigation of works like, random human motion detection, motion speed detection, trespasser detection, and many other applications, wherein human motion detection may be carried out passively from a remote monitoring station. Moreover, when using current system with multiple deployments at different locations, would eventually lead to a passive sensing system, analogous to sensor networks that transmits sensed information via nodes mounted at various location within a network. Thus, human recognition is possible under the domain of a passive sensing system.

\section{A. Research Contribution}

The main contributions of this paper are listed below:

- The study proposes a testbed for recognizing humans in indoor environments using two NI-USRPs, and it highlights the main challenges, experienced while setting up the testbed for the study.

- The study identifies possible setup for experiment, parameters that help in tuning of SDRs to the optimum level, along with setup to conduct further research in the domain of human recognition systems that may employ just single-input-single-output model of communication channel.

- The study provides a comparative analysis of two different machine learning models employed in this study for human identification. Moreover, the study accesses the level of accuracy of two different machine learning models that show an accuracy of $99 \%$ in identifying humans based on their patterns of locomotion.

The study is organized according to the following sections. Section II, provides a detailed background to the study. Section III provides a theoretical perspective, system model, and the method of data collection. Section IV describes the SVM based machine learning solution for pattern detection and classification. Section V provides a discussion on the study results and comparison of SVM performances. Finally, Section VI concludes the overall study.

\section{BACKGROUND}

Wireless signal propagation is influenced by various environmental factors, wherein wireless signal strength is mainly attenuated by multipath fading, path loss, and shadowing. Multipath results in a transmitted signal to arrive at the receiver, as multiple reflections of the original signal, from different paths, thereby causing severe distortion in the original signal component. Whereas the signal strength attenuates due to increased propagation distance and mainly relies on channel behavior, shadowing results in power loss due to physical objects appearing in signal propagation path.

The CSI of subcarrier [23], [24] frequencies show random variation, with an added distortion as a result of reflections in multipath propagation of a signal. Typically, random variations observed under normal conditions describe dynamics of CSI, whilst without the presence of nearby objects within the range. The CSI pertaining to various sub-carrier frequencies require methods of signal processing to de-noise and decompose the distinguishing features embedded in the signal, should be extracted for the purpose of motion pattern recognition. On the other hand, random human motion inevitably influences CSI elementary behaviour, and extracting meaningful information even becomes harder. Prior studies conducted explore the dynamic nature of RSS and extract patterns by applying machine learning algorithms on acquired data from multiple subcarriers. For example, [23], [24], [25] have applied Principal Component Analysis (PCA), [26] used Discrete Wavelet Transform (DWT), the CLEAN algorithm by [27], Doppler spectrum by [28] and even scale and time shift projections by [26], were used. Nonetheless, proposed signal processing and patterns recognition methods require high speed processing elements including scaled hardware resources that, in general, contribute to inefficient, unreliable and expensive methods of human recognition.

The random phase drift, as a result of sampling time offsets, is common in CSI phase measurements [27]. Consequently, for observed motion patterns, contrasting results can be seen with similar devices enabling CSI data generation. Processing devices with lower CSI-subcarrier sampling rate leads to processing delays and hence limits CSI based motion sensing in real time applications. Indoor environments include surrounding objects and motion observations may include background clutters. The Background elimination algorithm, for example by [27], subtracts static paths from the observed data, thus enables background clutter removal. While the likelihood criterion removes target reflection path from observed motion path, challenges in defining descriptor variables in noisy measurements still post serious concerns [28].CSI subcarriers in turn reflect random noise intensity levels. Each CSI sub-carrier component requires processing at an individual level, thus adding requirements of additional processing. 
MIMO systems [25] [27], on the other hand, employ multiple antennas in accretion to multiple receivers. Motion sensing systems based on MIMO, thus add increased device accessories and deployment costs. In addition, $5 \mathrm{GHz}$ CSI receivers implementing 114 and 132 sub-carriers for human motion sensing reveal only $94.0 \%$ accuracy [25], [24], meaning recognition accuracy is independent of added CSI channels. Here True Positive Rate (TPR) of CSI sub-carriers is independent, hence, added channels do not contribute to sensed motion recognition accuracy level.

As outlined in the previous sections, motion recognition based on CSI acquired from $\mathrm{WiFi}$, is process intensive, and requires a lot of resources for processing than processing only one radio carrier frequency. Therefore, this study proposes a single-input-single-output (SISO) communication model based human motion recognition, and the proposed system is evaluated using testbed setup employing two National Instruments universal radio peripherals (NI-USRPs) to discover patterns embedded in the dynamics of a radio signal. The optimal configuration settings of TX / RX subsystem are highlighted, along with the method of experimentation, and how to apply AI for classifying different human motion patterns to reveal identities of people, in particular four participants. The proposed system aims to show that human recognition is possible with only one radio carrier, which is far better than CSI based human recognition system that reveals human motion signatures based on multiple radio subcarrier frequencies.

\section{Methodology}

Our experimental setup was based on two NI-USRP [29] SDR devices. SDRs alleviate the hardware and software level tuning, and initial experimental setup was based on NI-USRP 2901 model and LabVIEW Communication Design Software [30]. For initial systematic trials, randomized control trials (RCT) based setup was used to search for the optimal alignment parameters as well as control configuration of the used SDRs. For data collection, four participants were employed to walk through a predefined path, whilst following the directions given prior to the conduct of trails. Using the developed software application, enabled collection of RSSI patterns that embedded human motion signatures, in the spreadsheet files having CSV format. Our experimental foundation relies on the following theoretical perspective, provides bases to software application development to capture human motion data.

\section{A. Transmitter-Receiver Sub-System}

Our testbed setup implements a Continuous Wave (CW) transmitter modulated with $10 \mathrm{kHz}$ sine-tone. Both the transmitter and receiver sub-system are equipped with one omnidirectional antenna (VERT2450) for transmission and reception respectively. For indoor environments, exhibit radio signal propagation characteristics wherein a transmitted signal converts to alias forms due to multipath propagation. In the current setup, transmitted $\mathrm{CW}$ arrives through multiple paths at receiver, and each different path adds delay and attenuation. CW transmission over longer distances, attenuates signal strength and wavelength considerably. In addition, RSS drastically varies due to small variations in multipath propagation, and variations equivalent to $5 \mathrm{~dB}$ in 1 minute have been observed in fixed receiver-transmitter pairs [31]. This study employs a fixed NI-USRP 2901 transmitter and receiver for effective results.

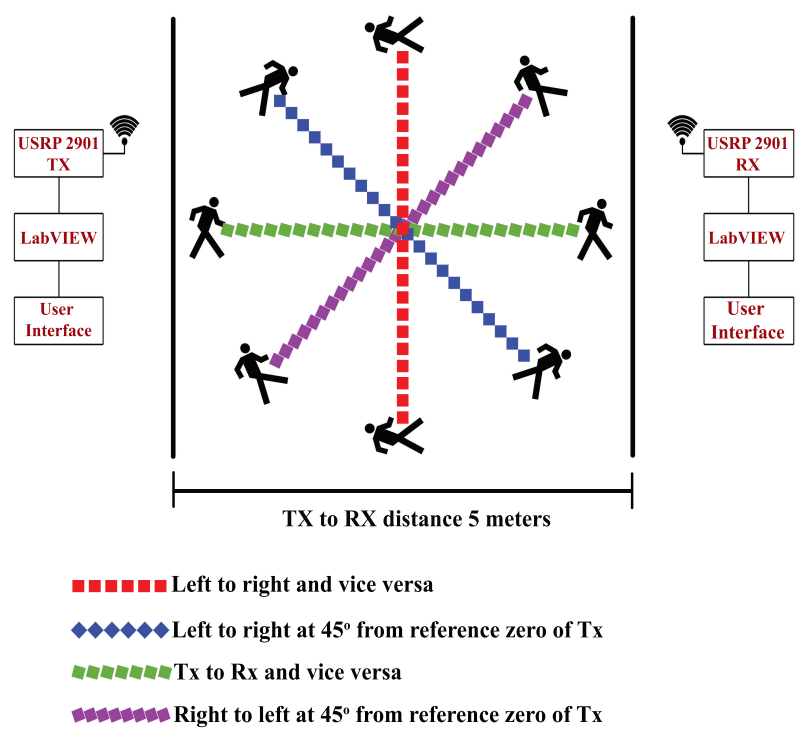

Fig. 1. Proposed System Model for Human Motion Patterns Recognition System.

CW signal strength arriving via mulipath at the receiver is given by the following relation:

$$
V=\sum_{k=1}^{N}\left\|V_{k}\right\| e^{-j \theta_{k}}
$$

where $V_{k}, \theta_{k}$ are the magnitude and phase of the $k$ th multipath component respectively. Symbol $N$ denotes total multipath components arriving at the receiver.

The NI USRP-2901 SDR model amplifies, down-converts, filters, digitizes, and decimates the received signal before the signal is transferred to the host computer. Similarly, the device up-samples, reconstructs, filters, up-converts, and amplifies the CW signal before its transmission into space. Testbed setup used two NI-USRP 2901 devices connected to two host computers. Fast Fourier Transform (FFT) is applied to extract frequency pilot signal in frequency domain. The demodulation process on the receiver recovers pilot signal strength, and the FFT provides the pilot RSS, which is expressed in decibel milliwatt $(\mathrm{dBm})$ and given by relation:

$$
\operatorname{RSS}(\mathrm{dBm})=10 \log _{10}\left(\|V\|^{2}\right)
$$

\section{B. System Model}

Our proposed system model is based on two NI-USRP devices and required a software application for data collection and processing. Two NI-USRP devices were set up with a LOS separation distance of 5 meters. Both devices were placed on computer tables with a height 1 meter above the ground. NI-USRPs devices are connected to host computers via USB 3.0 cabling, and custom developed LabVIEW application software enabled data collection. As depicted in Fig. 1, our proposed system model implements single input single output (SISO) channel. Clutters in the background are removed in this experiment, and the lab environment is completely an open space holding two tables placed opposite to each other, 


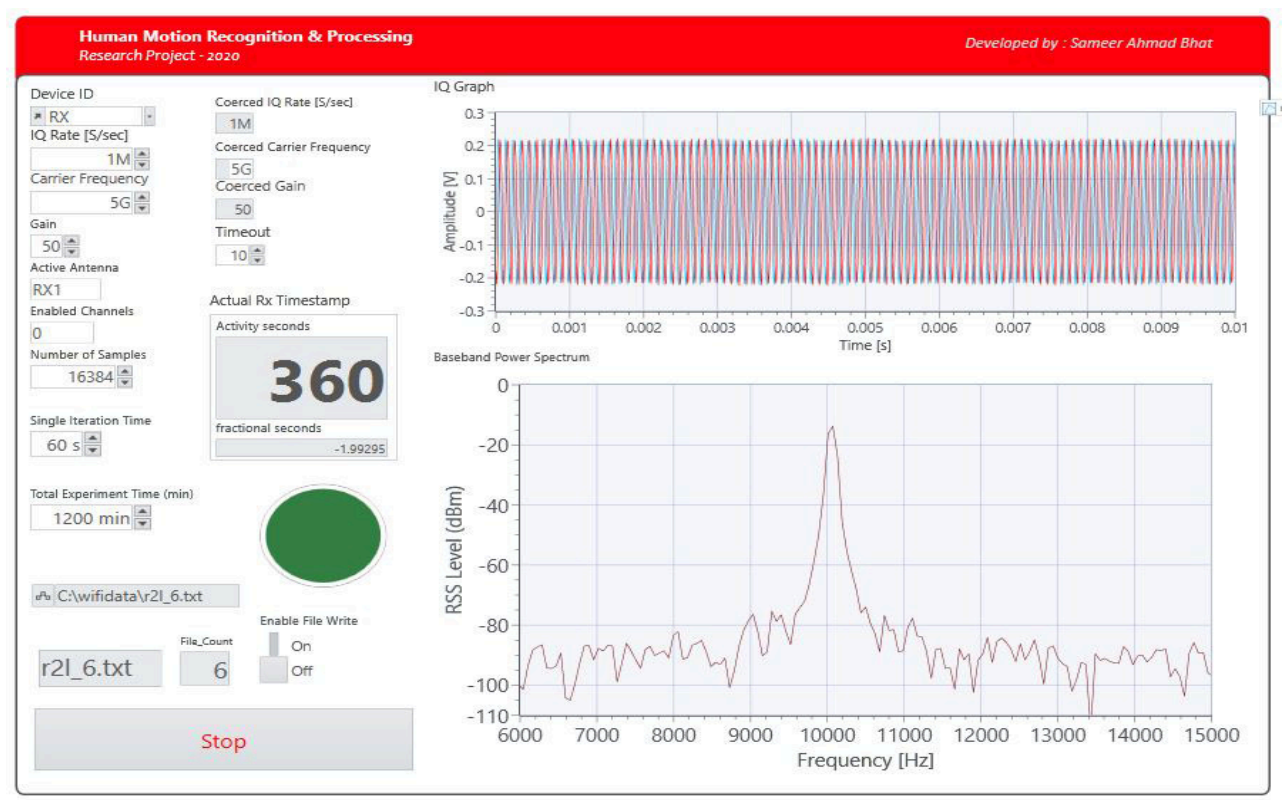

Fig. 2. Human Motion Recognition and Processing Application (HMRPA).

though a projector device is available in the room displaying the signal waveform on the side wall. Testbed setup using NI a single transmitter (TX) and receiver (RX) operate at $5 \mathrm{GHz}$ band. The proposed system model allows monitoring motion (walking) patterns in four different directions. Testbed setup was complete in the two phases - Software Application setup and Software application development,and both phases were sequentially carried out:

1) Phase I - Application Development: A human motion recognition and processing application (HMRPA) (see Fig. ??) was developed. Nex the developed software module was added to the NI-USRP RX application. Initially, the preliminary study testbed supported measurements and recordings of motion patterns for a single participant only. The HMPRA software module was integrated with the modified NI receiver application available in LabVIEW communication design software. HMPRA allowed parameter setting like experiment time, sampling, walking interval spacing and also the signal processing logic at baseband level. The HMRPA generated raw text files containing RSS samples of executed motion in one direction only. Subsequently, the Randomized Control Trial (RCT) experiment allowed collecting multiple motion patterns of a single person in four different directions (see Fig.1).

2) Phase II - Hardware Setup: Two NI-USRP 2901 SDRs were employed to set up the testbed. Both TX and RX applications were custom developed in LabVIEW IDE (Communications Design Software CDS)). NI-USRP set in transmitter mode, transmit a pilot tone of $10 \mathrm{KHz}$ at a carrier frequency of $5 \mathrm{GHz}$ using an omni-directional antenna. At the receiver, the RX application implemented a Fast Fourier Transform (FFT), with the Power Spectral Density (PSD) enabled estimations of recovered side-tone. The baseband Power Spectral Density (PSD) of RSS provided best possible TX - RX orientation, whilst having no obstacles in the line of sight (LoS).

With no motion LoS path, RSS stayed showed small deviations, even when a person staying 10 meters away from LoS. However, any participant walking through the LoS pilot tone drastically changed the pilot side-tone magnitude. Variations as such contained motion patterns samples, which were output in text files by the software application. Prior to testing, TX and $\mathrm{RX}$ were properly aligned to show maximum and stable pilot side-tone signal strength, and optimum results were observed with individual gains of both set to 50. RSS power levels observations at different gain settings for TX and RX (see Table II), and RCTs for control group RCT were carried out at room with zero human movements. RSS values here indicated nominal variations (see Fig. 3) at normal room temperature of $20^{\circ} \mathrm{c}$. Table I lists the recorded observations during orientation and alignment experiments.

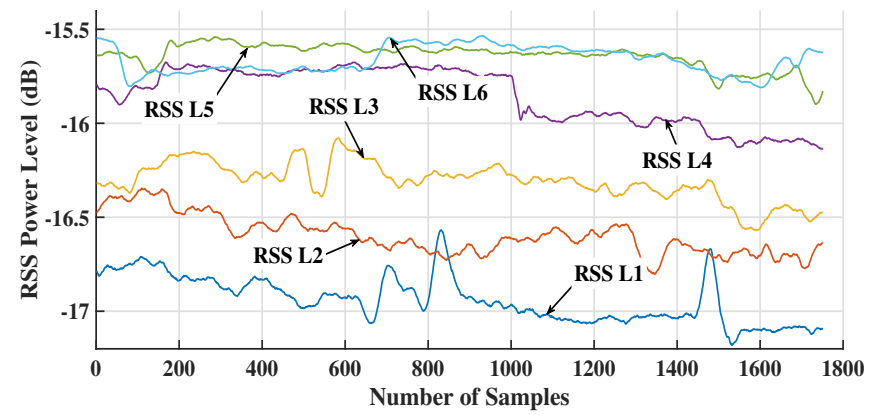

Fig. 3. Pilot RSSI Variations under Normal Conditions (Devoid of any Object in between TX and RX), and the "L" denotes Six observed levels of RSS. [32].

The NI-USRP based TX and RX orientation results revealed that TX and RX pair, with individual gains set to 50, show an optimal transmission characteristics at $0^{\circ}$, with both the devices placed one meter $(1 \mathrm{~m})$ above the ground level. The RSS drastically reduced to the minimum level at alternate orientations, and only maximized at 0 degrees. Therefore, LOS orientation of TX and RX established a direct communication link for our system model. 
TABLE I. ObSERVED VALUES FOR PROPER ORIENTATION OF TX AND RX

\begin{tabular}{|l|l|l|l|l|}
\hline $\begin{array}{l}\text { TX-RX } \\
\text { distance }\end{array}$ & $\begin{array}{l}\text { TX } \\
\text { Gain }\end{array}$ & $\begin{array}{l}\text { RX } \\
\text { Gain }\end{array}$ & $\begin{array}{l}\text { Orientation } \\
\text { (degrees) }\end{array}$ & RSS (dBm) \\
\hline $5 \mathrm{~m}$ & 50 & 50 & 0 & $-15 \mathrm{dBm}$ \\
\hline & & & 90 & $-29 \mathrm{dBm}$ \\
\hline & & & 180 & $-36 \mathrm{dBm}$ \\
\hline & & & 270 & $-34 \mathrm{dBm}$ \\
\hline $5 \mathrm{~m}$ & 50 & 40 & 0 & $-25 \mathrm{dBm}$ \\
\hline & & & 90 & $-33 \mathrm{dBm}$ \\
\hline & & & 180 & $-44 \mathrm{dBm}$ \\
\hline & & & 270 & $-42 \mathrm{dBm}$ \\
\hline $5 \mathrm{~m}$ & 30 & 0 & $-31 \mathrm{dBm}$ \\
\hline & 50 & & 90 & $-42 \mathrm{dBm}$ \\
\hline & & & 180 & $-52 \mathrm{dBm}$ \\
\hline & & & 270 & $-51 \mathrm{dBm}$ \\
\hline $5 \mathrm{~m}$ & & 20 & 0 & $-43 \mathrm{dBm}$ \\
\hline & 50 & & 90 & $-54 \mathrm{dBm}$ \\
\hline & & & 180 & $-66 \mathrm{dBm}$ \\
\hline & & & 270 & $-63 \mathrm{dBm}$ \\
\hline $5 \mathrm{~m}$ & & 10 & 0 & $-53 \mathrm{dBm}$ \\
\hline & 50 & & 90 & $-65 \mathrm{dBm}$ \\
\hline & & & 180 & $-75 \mathrm{dBm}$ \\
\hline & & & 270 & $-73 \mathrm{dBm}$ \\
\hline
\end{tabular}

TABLE II. OptimAL GAIN SETTING FOR TX AND RX

\begin{tabular}{|l|l|l|l|}
\hline TX and RX distance & TX Gain & RX Gain & RSS (dB) (Noise) \\
\hline $5 \mathrm{~m}$ & 0 & 0 & $-115 \mathrm{~dB}$ \\
\hline $5 \mathrm{~m}$ & 0 & 50 & $-78 \mathrm{~dB}$ \\
\hline $5 \mathrm{~m}$ & 50 & 0 & $-68 \mathrm{~dB}$ \\
\hline $5 \mathrm{~m}$ & 50 & 50 & $-15 \mathrm{~dB}$ \\
\hline
\end{tabular}

3) Dataset distribution: Two individual datasets collected for each participant enabled creation of Training and Test datasets. Both the datasets contained 10872 data samples acquired during 90 seconds. Thus, for four participants, the train CSV file contained 43488 samples. However, sequentially placed moves in the test set were used for estimating the classification accuracy of the selected ML model. The raw datasets were transformed into Comma-separated values (CSV) files since the ML model in LabVIEW required CSV type input. All the data sets were class labelled manually, and each dataset contained six numbers corresponding to six different classes of moves. Next, human motion data in CSV files train and test, were input to the SVM classifier of LabVIEW for evaluating the training and test accuracies respectively.

4) Data cleansing and anomaly correction: During the experiment, high frequency random noise was observed in all the four collected datasets. To filter out unwanted noise and glitches, a low pass filter and two consecutive moving average filters with a window size of 50 were applied on datasets. Effects of removing high frequency components and random noise can be seen in Fig. 4 and Fig. 5, with Fig. 4 showing a move set with added random noise, whereas a cleansed dataset can be observed in Fig. 5 after applied filtering. Abreast removing noise content, unusual movements such as hand gestures, or leaning backwards on a wall, or turning around for the next move, were observed, however, these inconsistencies were manually removed by overwriting the unwanted samples with mean variations.

For each move of each subject, RSS motion patterns embedded distortion and severe noise components. Each motion signature also showed anomalies resulting in trends in the data patterns. RSS strength drastically reduced with taller participants, leading to a change in scale of measurement. Thus, before RSS data was input to the SVM algorithm, processing was carried out at an earlier stage. Data processing

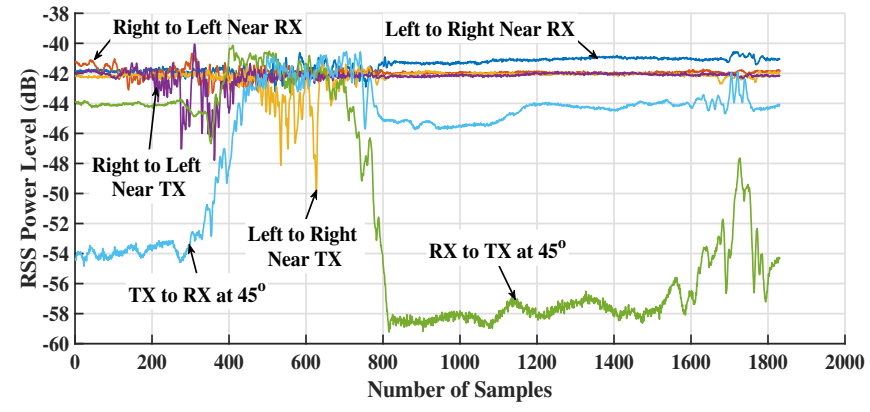

Fig. 4. Raw Movement Patterns before Filtering.

involved computing mean, standard deviations, detrending, normalization, and windowing to extract the required motion pattern only. Fig. 9 to 12, depict the extracted motion patterns. Notice some motion signatures contain unusual variations ranging over the last 300 samples. Variations as such represent participants movements such as turning around, raising arm, and leaning. These undesired data variations were manually removed, and finally cleaned motion signatures were acquired for human motion predictive analysis.

\section{Human Classification Using Motion SIGNATURES}

The study classifies humans based on identified unique motion patterns observed in variations revealed by pilot signal. Recognizing and classifying human motion requires designating a class to each unique move dataset of each participant. Therefore, machine learning based motion recognition and classification algorithms are realized. Motion patterns observed from pilot signal variations exhibit nonlinear behavior, and Support Vector Machine (SVM) algorithms are mostly applicable to such applications, enabling exploration of hidden patterns in linear as well as non-linear data [33]. SVMs employ support vectors set out of training data to classify any unknown data sample $q$ by comparing given input samples against the support vectors:

$$
\operatorname{sign}\left(\sum y_{i} \alpha_{i} K\left(p_{i}, q\right)+b\right)
$$

where $y_{i}$ represents class association $(-1$ or +1$) ; \alpha_{i}$ is the weight coefficient or Lagrange multiplier; $K$ is the kernel function; $p_{i}$ is the support vector data; $i$ is the index from $i=1,2,3, \ldots l$; and $b$ represents the hyperplane distance from the origin. Next, subsections outline the variations of SVM algorithms.

\section{A. SVM - Multiple Class input Categorization}

Classification using the SVM algorithm typically requires defining a minimum of two classes or categories. Classes exceeding more than two, directs SVM algorithms to implement a one-versus-one approach for generating a binary classification model that corresponds to every possible class combination. Abreast, SVM algorithm implements polling method to derive most suitable class for a known input. For multiple classes resulting out of a polling method, the algorithm determines the class nearest to the sampled input. Thus, for the $s$ number of classes generates $s \times(s-1) / 2$ classification models, enabling a contrast in each category of input data. 


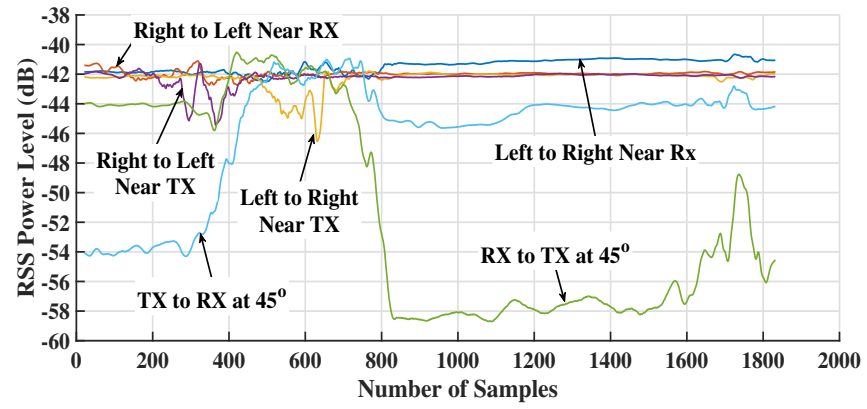

Fig. 5. Movement Patterns after Filtering.

\section{B. Optimum Model Selection}

SVM model determines classification of data samples, and classification problems involving single and multiple classes in the input data, employ either one-class model or multi-class models, known as C-SVC or nu-SVC.

1) SVM - Type C-SVC: To minimize the estimated error function, C-SVC model targets segregation of data samples separated by close or narrow margins using trained C-SVC model:

$$
\min _{z, b, \xi} \frac{1}{2} z^{T} z+\alpha \sum_{i=1}^{l} \xi_{i}
$$

Subject to

$$
y_{i}\left(z^{T} K\left(p_{i}\right)+b\right) \geq-\xi_{i} ; \quad \xi_{i} \geq 0, \quad i=1,2, \ldots l
$$

where $z, \alpha$ and $\xi$ represent normal vector of the hyperplane to origin, cost parameter, and the slack variable respectively. $\alpha$ - the Cost parameter uses partial training errors to define new soft margins subject to SVM algorithm failing to set out a an optimized margin. Selecting high values of cost parameter enables partial error removal, thereby resulting in a narrower margin and perfect classifications.

2) $S V M$ - Type $N u$-SVC: SVM class comparisons using $\mathrm{Nu}$-SVC model enable precise controlling of training errors and support vectors set, using a parametric control called $n u$. $\mathrm{Nu}-\mathrm{SVC}$ model requires training with input data to minimize the error function:

$$
\min _{z, b, \xi} \frac{1}{2} w^{T} w-v \lambda+\frac{1}{l} \sum_{i=1}^{l} \xi_{i}
$$

Subject to

$$
Y_{i}\left(z^{T} K\left(x_{i}\right)+b\right) \geq \rho-\xi_{i} ; \xi_{i} \geq 0, i=1,2, \ldots l ; \lambda \geq 0
$$

where $v$ and $\xi$ represent $n u$ parameter and the slack variable, respectively. $n u(0 \leq n u \leq 1)$ parameter specifies maximum training errors ratio and minimum support vector count corresponding to sample count. Abreast increasing acceptance of texture defects, high value to $n u$ increases probability of acceptance of texture dissimilarities.

The C-SVC classification model is used and a multifeatured vector data sets of four participants with associated label sets is prepared. During training, training data is manually labelled, however, testing determines the classes from the model itself.

\section{Kernels}

The SVM classifier comes with different kernel types. One of the kernels is categorized as a linear classifier, generally implements a linear kernel as a product of the input sample feature vector times the sample support vector, however, SVMs also support non-linear type of classifiers. Table III shows the most commonly used nonlinear kernels in SVM classifiers.

TABLE III. KERNEL TYPES [34]

\begin{tabular}{|l|l|}
\hline Kernel type & Model Equation \\
\hline Linear & Kernel $\left(x_{i}, x\right)$ \\
\hline Polynomial & $\left(\gamma \times \operatorname{Kernel}\left(x_{i}, x\right)+\right.$ Coefficient $)$ \\
\hline RBF & $e^{-\gamma\left\|x_{i}-x\right\|^{2}}$ \\
\hline Sigmoid & $e^{-\frac{\left\|x_{i}-x\right\|^{2}}{2 \times \sigma^{2}}}$ \\
\hline
\end{tabular}

\section{Feature Extraction}

Feature extraction required preparing feature vectors out of the collected pilot signal variation datasets of all the four participants. The extracted feature vector space consisted of RSS variations for each participant observed in four directions, including features such as mean, variance, standard deviation, and skewness. For four participants, datasets $\left(p_{i}^{1}, q_{j}^{1}\right),\left(p_{i}^{2}, q_{j}^{2}\right),\left(p_{i}^{3}, q_{j}^{3}\right)$ and $\left(p_{i}^{4}, q_{j}^{4}\right)$ were collected, processed and then manually assigned class labels 1,2,3 and 4, corresponding the to four different participant, respectively (superscripts and superscripts denote index of participant and collected data points, respectively). The extracted feature vectors can be expressed in matrix as:

$$
\left(\begin{array}{cccccc}
\left(p_{1,1}^{1}\right) & \left(p_{1,2}^{1}\right) & \left(p_{1,3}^{1}\right) & \cdots & \left(p_{1, n}^{1}\right) & \rightarrow\left(q_{1}^{1}\right) \\
\vdots & \vdots & \vdots & \vdots & \vdots & \vdots \\
\left(p_{i, 1}^{1}\right) & \left(p_{i, 2}^{1}\right) & \left(p_{i, 3}^{1}\right) & \cdots & \left(p_{i, n}^{1}\right) & \rightarrow\left(q_{1}^{1}\right) \\
\hline\left(p_{1,1}^{2}\right) & \left(p_{1,2}^{2}\right) & \left(p_{1,3}^{2}\right) & \cdots & \left(p_{1, n}^{2}\right) & \rightarrow\left(q_{2}^{2}\right) \\
\vdots & \vdots & \vdots & \vdots & \vdots & \vdots \\
\left(p_{i, 1}^{2}\right) & \left(p_{i, 2}^{2}\right) & \left(p_{i, 3}^{2}\right) & \cdots & \left(p_{i, n}^{2}\right) & \rightarrow\left(q_{2}^{2}\right) \\
\hline\left(p_{1,1}^{3}\right) & \left(p_{1,2}^{3}\right) & \left(p_{1,3}^{3}\right) & \cdots & \left(p_{1, n}^{3}\right) & \rightarrow\left(q_{3}^{3}\right) \\
\vdots & \vdots & \vdots & \vdots & \vdots & \vdots \\
\left(p_{i, 1}^{3}\right) & \left(p_{i, 2}^{3}\right) & \left(p_{i, 3}^{3}\right) & \cdots & \left(p_{i, n}^{3}\right) & \rightarrow\left(q_{3}^{3}\right) \\
\hline\left(p_{1,1}^{4}\right) & \left(p_{1,2}^{4}\right) & \left(p_{1,3}^{4}\right) & \cdots & \left(p_{1, n}^{4}\right) & \rightarrow\left(q_{4}^{4}\right) \\
\vdots & \vdots & \vdots & \vdots & \vdots & \vdots \\
\left(p_{i, 1}^{4}\right) & \left(p_{i, 2}^{4}\right) & \left(p_{i, 3}^{4}\right) & \cdots & \left(p_{i, n}^{4}\right) & \rightarrow\left(q_{4}^{4}\right)
\end{array}\right)
$$

Where $i$ represents a sample index taking value $i=1 \rightarrow 1812$ for each subset matrix. $j=1 \rightarrow 4$. $p_{1,1}^{1}$ to $p_{i, n}^{1}$ represent a subset matrix representing a dataset comprising pilot samples $p^{1}$ including assigned label vector $q^{1}$ for each participant. Similarly, three participants' feature vectors $p_{1,1}^{2}$ to $p_{i, n}^{2}, p_{1,1}^{3}$ to $p_{i, n}^{3}$ and $p_{1,1}^{4}$ to $p_{i, n}^{4}$ are designated labels $q_{2}^{2}, q_{3}^{3}$ and $q_{4}^{4}$, respectively.

\section{E. Training - SVM Model}

The SVM model was generated using the randomized optimization algorithm (ROA) available in LabVIEW. ROA running on the laptop equipped with Intel Core I3 processor and $8 \mathrm{~GB}$ of RAM, enabled fine tuning and optimization of SVM model parameters. After 250 iterations, the generated SVM model depicted approximately $99 \%$ training accuracy. 
Observed training results on selected kernel types are given in Table IV. Both C-SVC and Nu-SVM models showed comparative results, however, SVM model of type C-SVC using Radial Bias Function (RBF) kernel resulted in nominal parameter settings with highest efficiency among all (see Table IV entry at row 3). With lowest prediction error against, the competitors (linear, polynomial, and sigmoid) guided us to select the CSVC model with RBF kernel type, for this study. Results in Table IV explain that the RBF kernel function shows the lowest classification error. Fig. 6 to 9 depict each participants' raw motion pattern datasets, embedded with unwanted noise components, emerging as a result of pilot signal variations.

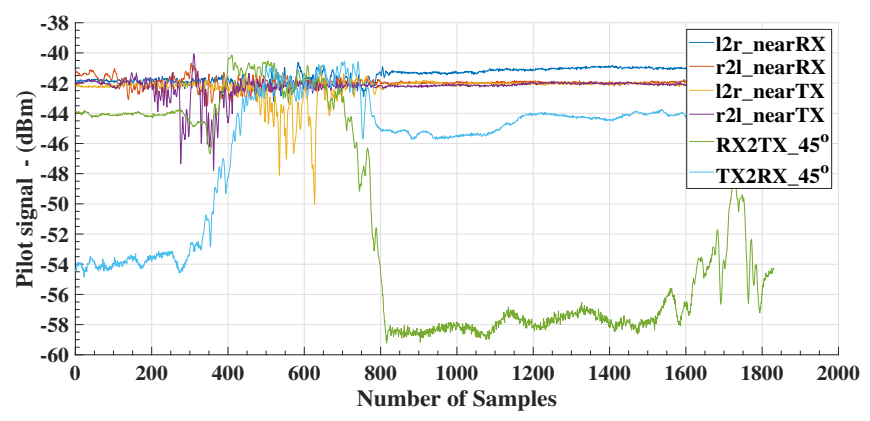

Fig. 6. Raw Motion Patterns of Participant -1 .

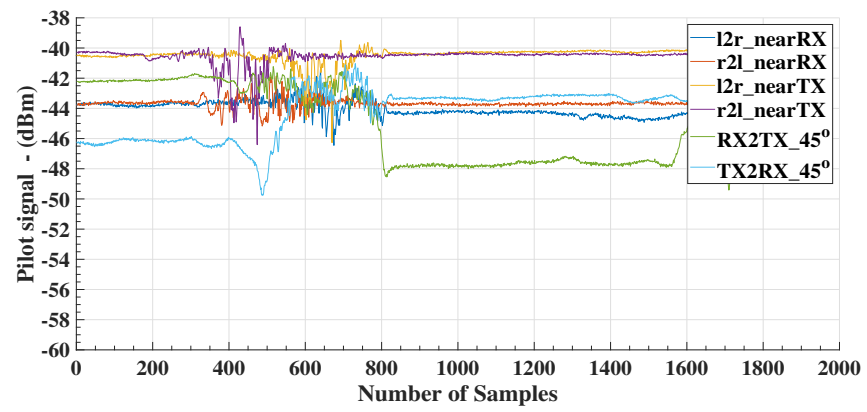

Fig. 7. Raw Movement Patterns of Participant -2 .

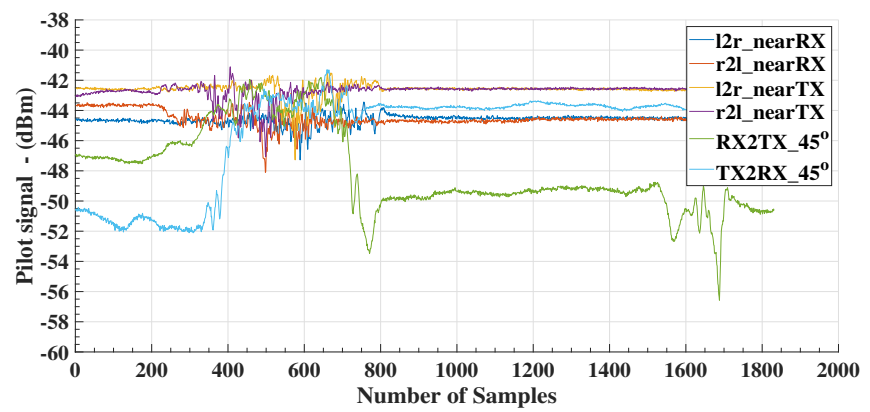

Fig. 8. Raw Movement Patterns of Participant - 3 .

\section{F. Prediction - Testing the SVM Model}

Test datasets prepared initially, were used to test the prediction accuracy of trained SVM models. Test set contained sequentially arranged moves, however, without class labels this time, and using an overall test dataset containing $1812 \times 4=7248$ (in each feature vector) samples in total allowed performance measurements. The overall test data set

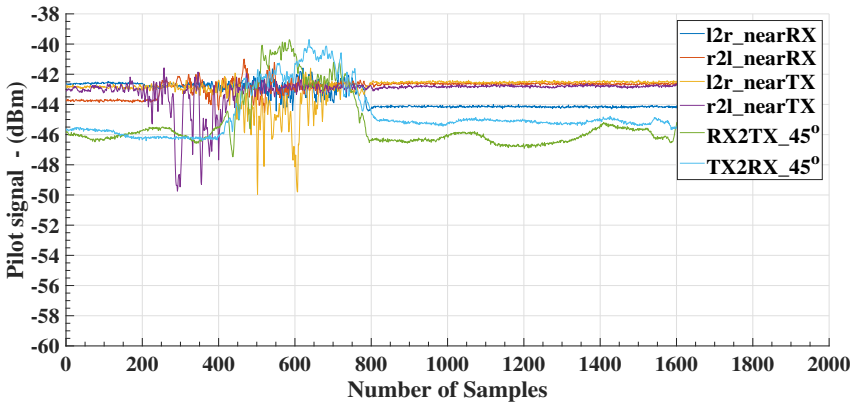

Fig. 9. Raw Movement Patterns of Participant -4 .

was used to measure the performance of the SVM model, which resulted in predicting each participant's identity with an accuracy of approximately 98\%. Fig. ?? shows the screenshot of a human prediction application developed in LabVIEW, and the upper plot here shows four individual motion patterns input to the SVM algorithm, whereas lower plot shows the prediction results. The prediction result indicates some deviations in estimating accuracy of some samples. Abreast using the overall test dataset, each participant's motion dataset were isolated, and then tested using the trained SVM model individually on each participant's motion dataset. Here, the SVM model predicted each participant's identity with an accuracy of nearly 99\%. Fig. 10 illustrates the True Positive Rate (TPR) and False Positive Rate (FPR) accuracies observed on the overall dataset containing moves of all the participants.
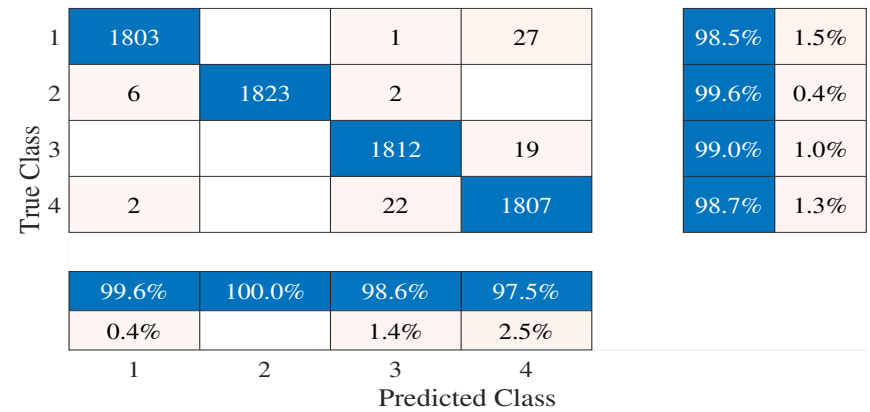

Fig. 10. Confusion Matrix of Overall Prediction Results.

\section{RESUlts AND Discussion}

Our testbed setup revealed numerous challenges, particularly with the lower strength of the pilot signal. The NI-USRP 2901 RX, showed that the pilot signal is highly sensitive to ambient variations, and hence considerable variations can be observed in the pilot RSS, having fine human movements, such as turning of head, raising arm or speaking. Our experimental setup ensured zero human motion, during the installation and initial testing. Fig. 3 shows the pilot signal strength variations, recorded under $27^{\circ}$ room temperature, with $\mathrm{Tx}$ and $\mathrm{RX}$ gains both set to 70 . The six of the pilot signal variations, show how pilot RSS varies at the nominal room temperature, with zero human presence.

The pilot signal variations range between $-15.4 \mathrm{~dB}$ to $17.5 \mathrm{~dB}$ approximately. When the testbed was exposed to detect the human movements, the pilot RSS showed drastic variations, mostly with a power level below $-45 \mathrm{dBm}$, thereby presenting unique motion patterns via pilot signal. Thus, the 
TABLE IV. A Randomized SEARch Optimization Criteria Evaluated in Lab VIEW

\begin{tabular}{|c|c|c|c|c|c|c|c|c|}
\hline Sno. & kernel & svm_type & c & nu & deg & gamma & coeff & accu \\
\hline 1 & RBF & CSV-C & 0.7 & 0 & 3 & 0.7 & 0 & 0.999 \\
\hline 2 & $\mathrm{RBF}$ & CSV-C & 0.9 & 0 & 3 & 0.4 & 0 & 0.999 \\
\hline 3 & RBF & CSV-C & 0.8 & 0 & 3 & 0.7 & 0 & 0.999 \\
\hline 4 & RBF & CSV-C & 1 & 0 & 3 & 0.6 & 0 & 0.999 \\
\hline 5 & RBF & CSV-C & 1 & 0 & 3 & 0.7 & 0 & 0.999 \\
\hline 6 & Polynomial & CSV-C & 0.4 & 0 & 3 & 0.4 & 1 & 0.998 \\
\hline 7 & Polynomial & CSV-C & 0.9 & 0 & 8 & 0.1 & 1 & 0.998 \\
\hline 8 & Polynomial & CSV-C & 0.8 & 0 & 4 & 0.1 & 1 & 0.998 \\
\hline 9 & Polynomial & CSV-C & 1 & 0 & 3 & 0.7 & 1 & 0.998 \\
\hline 10 & Polynomial & CSV-C & 0.7 & 0 & 2 & 0.7 & 1 & 0.998 \\
\hline 11 & Polynomial & CSV-C & 0.5 & 0 & 4 & 0.5 & 1 & 0.998 \\
\hline 12 & Polynomial & CSV-C & 1 & 0 & 2 & 0.6 & 1 & 0.998 \\
\hline 13 & Polynomial & CSV-C & 0.7 & 0 & 9 & 0.1 & 1 & 0.998 \\
\hline 14 & Polynomial & CSV-C & 0.2 & 0 & 3 & 0.8 & 1 & 0.998 \\
\hline 15 & Polynomial & CSV-C & 0.9 & 0 & 2 & 0.6 & 1 & 0.998 \\
\hline 16 & $\mathrm{RBF}$ & CSV-C & 0.4 & 0 & 3 & 0.7 & 0 & 0.998 \\
\hline 17 & Polynomial & CSV-C & 0.2 & 0 & 4 & 0.7 & 1 & 0.998 \\
\hline 18 & Polynomial & CSV-C & 0.7 & 0 & 2 & 0.5 & 1 & 0.998 \\
\hline 19 & Polynomial & CSV-C & 0.6 & 0 & 6 & 0.2 & 1 & 0.998 \\
\hline 20 & Polynomial & CSV-C & 0.1 & 0 & 5 & 0.4 & 1 & 0.998 \\
\hline 21 & Polynomial & CSV-C & 0.7 & 0 & 3 & 0.8 & 0 & 0.998 \\
\hline 22 & Polynomial & CSV-C & 0.1 & 0 & 3 & 0.7 & 1 & 0.998 \\
\hline 23 & Polynomial & CSV-C & 0.4 & 0 & 4 & 0.6 & 1 & 0.998 \\
\hline 24 & Polynomial & CSV-C & 0.1 & 0 & 2 & 0.4 & 1 & 0.998 \\
\hline 25 & Polynomial & CSV-C & 0.4 & 0 & 7 & 0.1 & 1 & 0.998 \\
\hline 26 & Polynomial & CSV-C & 1 & 0 & 3 & 0.8 & 0 & 0.998 \\
\hline 27 & Polynomial & CSV-C & 0.8 & 0 & 4 & 0.6 & 1 & 0.998 \\
\hline 28 & Polynomial & CSV-C & 0.1 & 0 & 2 & 0.6 & 1 & 0.998 \\
\hline 29 & Polynomial & CSV-C & 0.6 & 0 & 6 & 0.1 & 1 & 0.998 \\
\hline 30 & Polynomial & CSV-C & 0.8 & 0 & 3 & 0.6 & 1 & 0.997 \\
\hline 31 & Polynomial & CSV-C & 0.6 & 0 & 3 & 0.7 & 0 & 0.997 \\
\hline 32 & Polynomial & CSV-C & 0.6 & 0 & 2 & 0.8 & 1 & 0.997 \\
\hline 33 & Polynomial & CSV-C & 0.4 & 0 & 3 & 0.4 & 0 & 0.997 \\
\hline 34 & Polynomial & CSV-C & 0.9 & 0 & 2 & 0.8 & 1 & 0.997 \\
\hline 35 & Polynomial & CSV-C & 0.1 & 0 & 3 & 0.6 & 0 & 0.997 \\
\hline 36 & Polynomial & CSV-C & 0.8 & 0 & 5 & 0.2 & 1 & 0.997 \\
\hline 37 & RBF & CSV-C & 0.2 & 0 & 3 & 0.4 & 0 & 0.997 \\
\hline 38 & Polynomial & CSV-C & 0.2 & 0 & 3 & 0.7 & 0 & 0.997 \\
\hline 39 & Polynomial & CSV-C & 0.9 & 0 & 4 & 0.5 & 1 & 0.997 \\
\hline 40 & Polynomial & CSV-C & 0.9 & 0 & 4 & 0.7 & 1 & 0.997 \\
\hline 41 & Polynomial & CSV-C & 1 & 0 & 4 & 0.7 & 1 & 0.996 \\
\hline 42 & Polynomial & CSV-C & 0.5 & 0 & 2 & 0.1 & 1 & 0.996 \\
\hline 43 & Polynomial & CSV-C & 1 & 0 & 4 & 0.5 & 1 & 0.996 \\
\hline 44 & Polynomial & CSV-C & 0.6 & 0 & 2 & 0.1 & 1 & 0.996 \\
\hline 45 & Polynomial & CSV-C & 0.1 & 0 & 3 & 0.3 & 0 & 0.996 \\
\hline 46 & Polynomial & CSV-C & 1 & 0 & 6 & 0.3 & 1 & 0.996 \\
\hline 47 & Polynomial & CSV-C & 0.5 & 0 & 5 & 0.8 & 1 & 0.996 \\
\hline 48 & Polynomial & CSV-C & 0.5 & 0 & 4 & 0.7 & 0 & 0.996 \\
\hline 49 & Polynomial & CSV-C & 0.5 & 0 & 5 & 0.7 & 1 & 0.996 \\
\hline 50 & Polynomial & CSV-C & 0.9 & 0 & 3 & 0.2 & 0 & 0.996 \\
\hline
\end{tabular}

proposed human motion recognition and identification method is highly sensitive, and our analysis of collected results (see Fig. 12 to 17) suggests that fluctuations in pilot RSS due to human motion can be extracted and analyzed to uncover unique human motion patterns for recognizing a person's identity. Therefore, our study results are applicable to environments such as smart authentication systems, or patient monitoring systems, and healthcare monitoring systems, wherein passive sensing is mostly preferred over traditional methods, typically based on active sensing devices.

1) Comparative Analysis: SVM algorithm prediction accuracy was estimated on four different kernel functions - linear, radial basis function (RBF), sigmoid and polynomial. Using grid-search optimization, setting different input parameters, as required by the kernel functions, enabled the prediction model to achieve maximum classification accuracy level [35]. Table IV defines the first 50 configuration parameters used for tuning the SVM prediction model. As shown in the Table IV, the SVM type C-SVC models using a polynomial kernel reveals the most efficient hyperplane model. The CSVC kernel with RBF kernel implemented in labVIEW showed
99.9\% training accuracy, whereas classification accuracy of human identification (participant identity prediction) on the train dataset was observed to be approximately $98 \%$ with polynomial kernel. CSV-C with sigmoid type kernel is strongly competitive with the other CSV-C types, however, resulting in a prediction accuracy of just below 95\%. Fig. ?? and ?? show the probability distribution of training accuracy levels realized against the different kernels and SVM model types respectively. The CSV-C type $\mathrm{RBF}$ kernel is observed to deliver higher prediction accuracy than other kernel types. CSV-C with linear kernel was realized to be least efficient as compared to the sigmoid kernel, which showed a mean accuracy level at $91 \%$.

Estimation accuracy of linear kernel shows the least probability distribution, while the sigmoid shows $83 \%, 87 \%, 91 \%$, $94 \%$ and $97 \%$, for minimum, lower quartile, median, upper quartile and maximum distributions respectively. Among all the four, the polynomial kernel spans lower distribution, and shows lower and upper quartiles at $95 \%$ and $98 \%$ respectively, affirming that polynomial has considerable training accuracy compared to the RBF kernel with overlapped upper and lower 


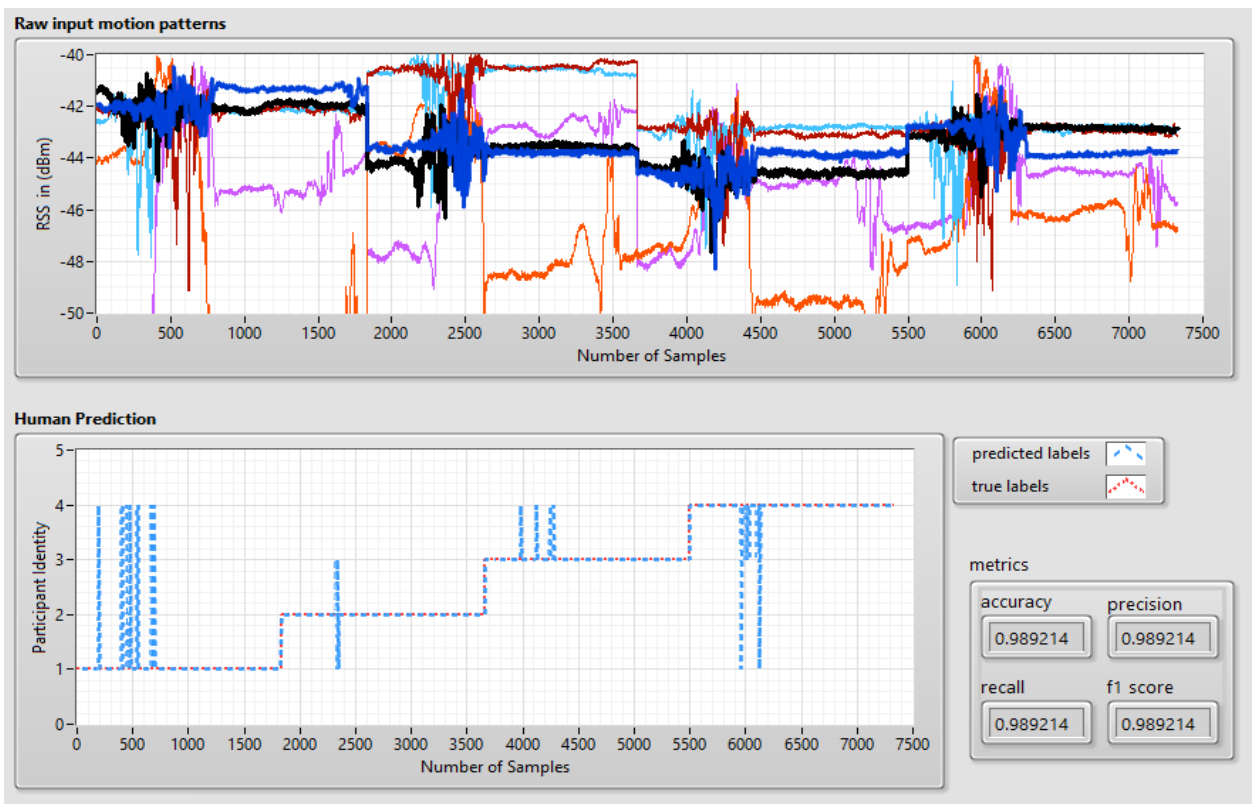

Fig. 11. LabVIEW: SVM Prediction Results on Test Dataset of all Participant.
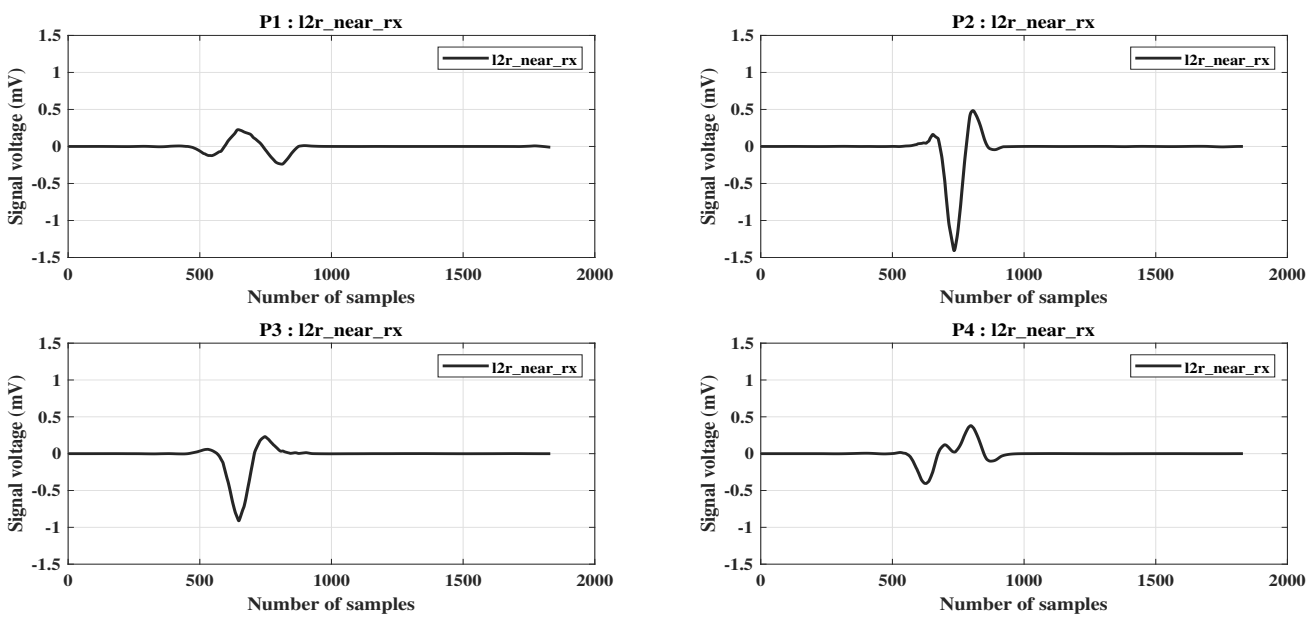

Fig. 12. Processed Move(1) Motion Signatures.

quartiles. On the other hand, sigmoid kernel has a more wider distribution, spanning approximately from $88 \%$ to $95 \%$.

Observing the boxplot of SVM model types, describes higher efficiency of CSV-C on non-linear human motion dataset with lower and upper quartiles, both above the $95 \%$ accuracy level. Although Nu-SVC showed wider accuracy distribution than its counterpart CSV-C, the observed accuracy range on the non-linear human motion dataset is still lower than CSV-C, with both lower and upper quartiles below the mean of CSV-C type kernel. This clearly shows the CSV-C is suitable for applications involving non-linear data distributions, in particular human motion recognition using highly sensitive pilot signal RSS.

2) Testbed requirements: NI-USRP devices may use different hardware clock signal generators. Often this raises critical concerns since mismatch in clock signal frequency can result in the receiver showing incorrect data. The NI-USRP devices employed by this study, showed deviating results due to mismatch in their clock signals. Since the devised system needed recognizing motion patterns in microsecond time, the USRPs experienced jitter including pilot signal frequency drift to $\pm 4 \mathrm{KHz}$. Thus, pilot signal appeared $10 \mathrm{KHz} \pm$ $4 \mathrm{KHz}$, and to abase frequency offsets in the pilot signal, manually changing sampling frequency in NI-USRP devices enabled tuning the receiver (RX) to show correct pilot signal frequency response, which in our case was $10 \mathrm{KHz}$. Thus, tuning of both the TX/RX parameters, such as transmitted power, sampling frequency, pilot signal frequency, distance and receiver orientation, ensured optimal system behaviour. Although sampling rate is adjusted manually in this study, use of an external reference clock generator is recommended for future studies.

Moreover, this study employed NI-USRP 2901, connected 

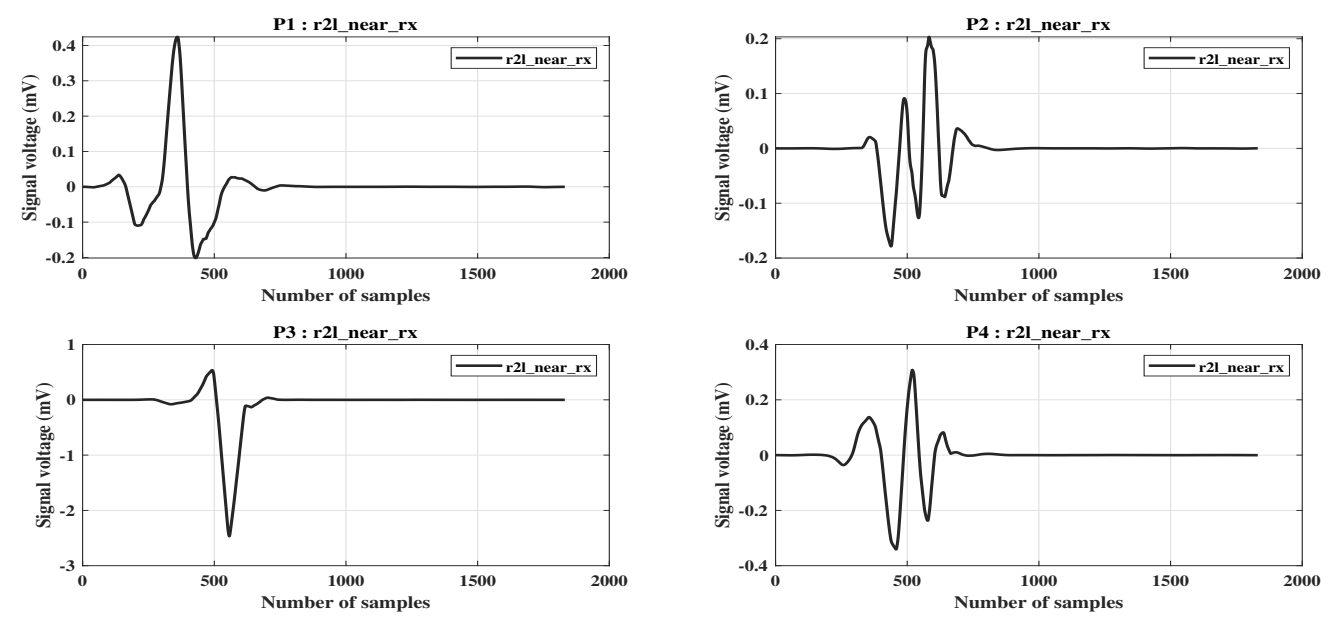

Fig. 13. Processed Move(2) Motion Signatures.
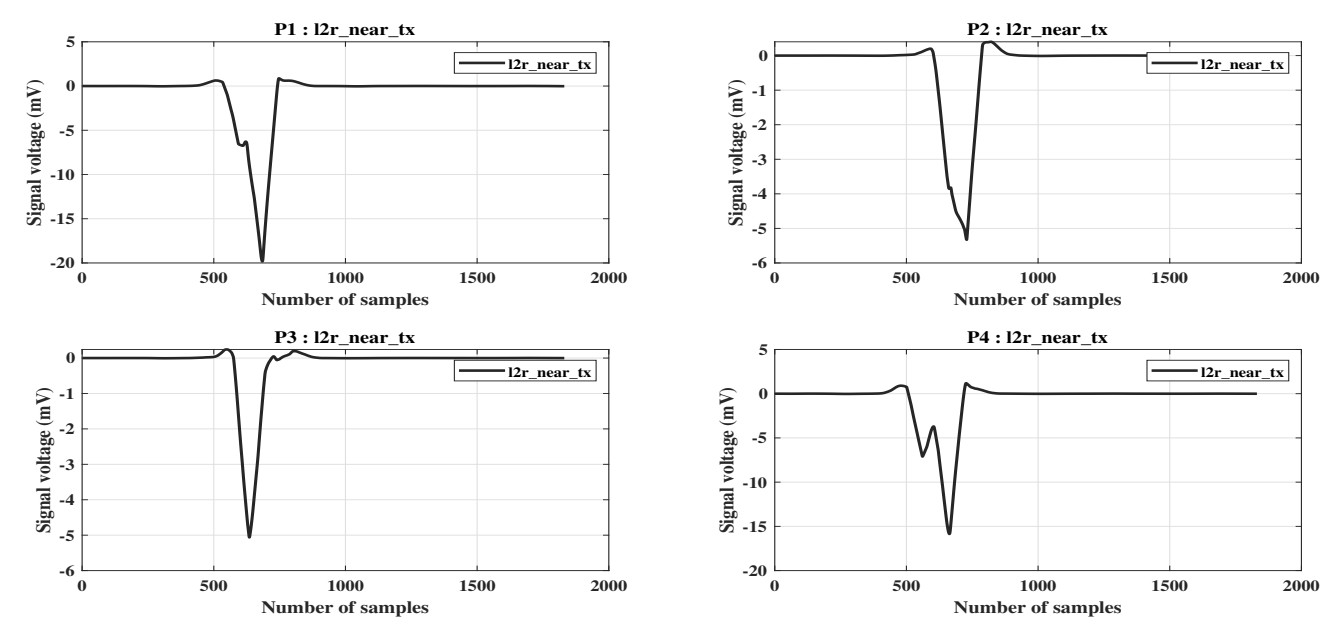

Fig. 14. Processed Move(3) Motion Signatures.
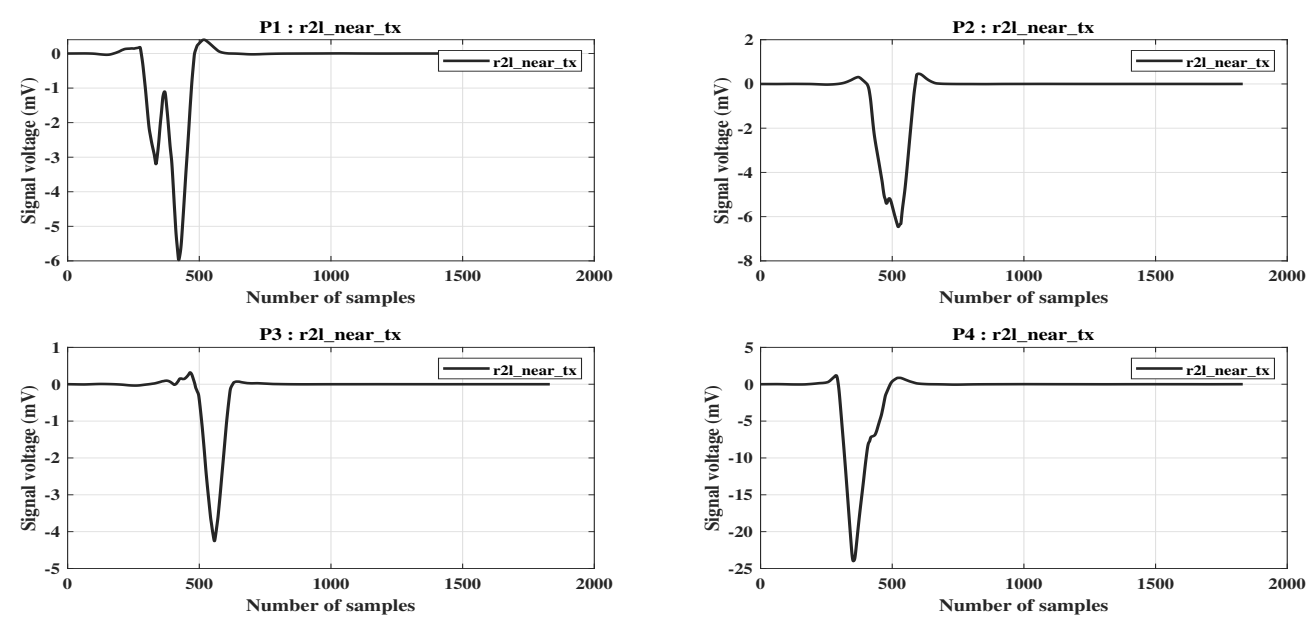

Fig. 15. Processed Move(4) Motion Signatures. 

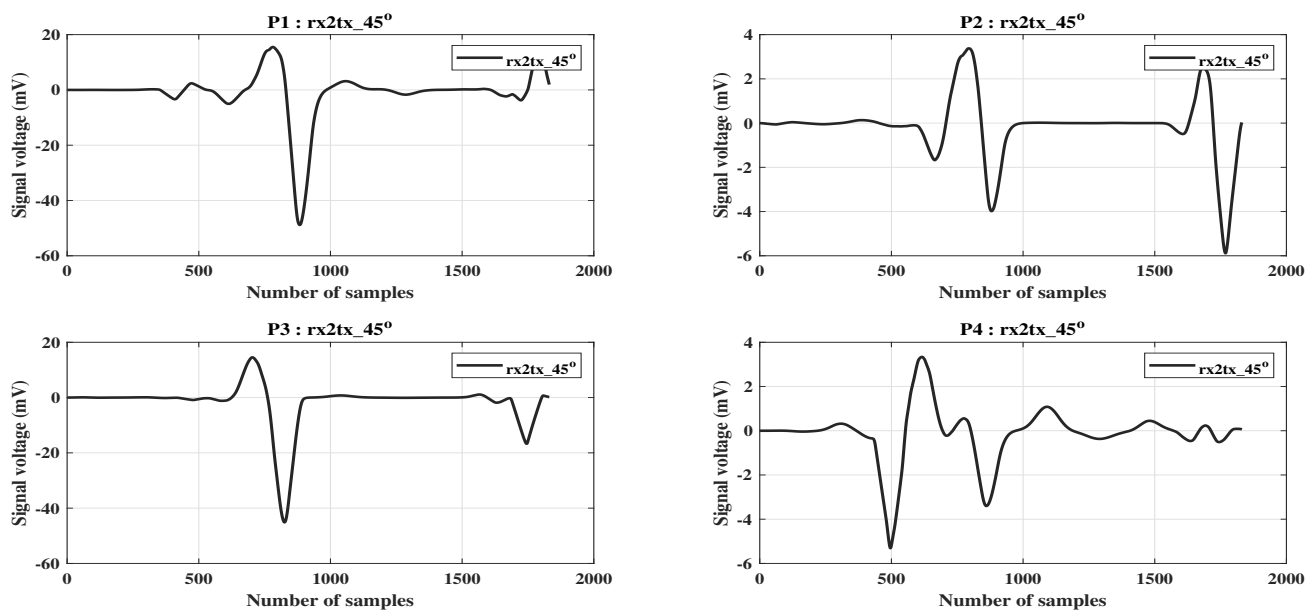

Fig. 16. Processed Move(5) Motion Signatures.
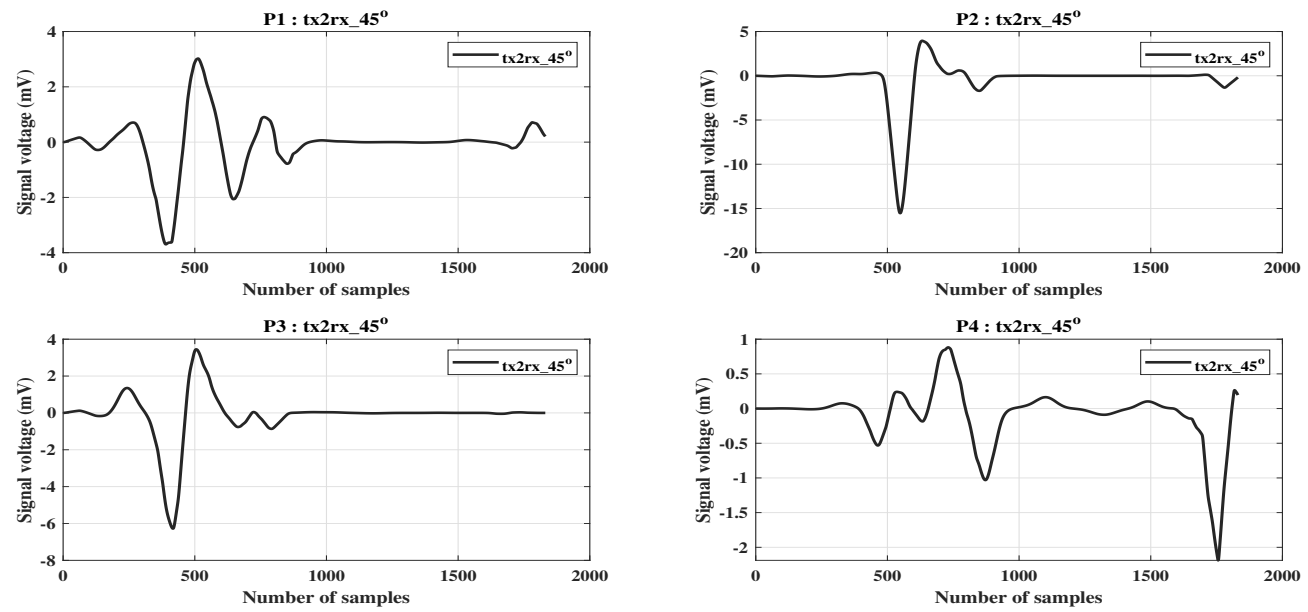

Fig. 17. Processed Move(6) Motion Signatures.

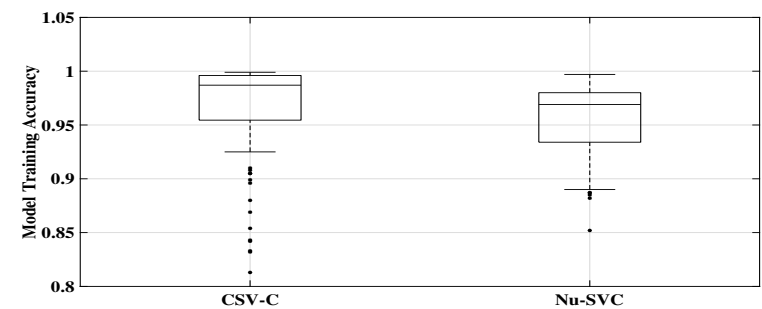

Fig. 18. Boxplot of Training Accuracy versus Kernel Types.

to a host computer via USB-3.0 port. Despite the high speed access offered by the USB-3.0 communication protocol and wideband capabilities (from $70 \mathrm{MHz}$ to $6 \mathrm{GHz}$ ) of NIUSRP 2901, the LabVIEW communication design software application posed start up delays, and even during the device initialization phase, often lag to communicate with the host device, the NI-USRP. Therefore, for future research work, high speed devices such as NI-USRP 2921 [30], offering 1 Gigabit Ethernet speeds is highly recommended since real time

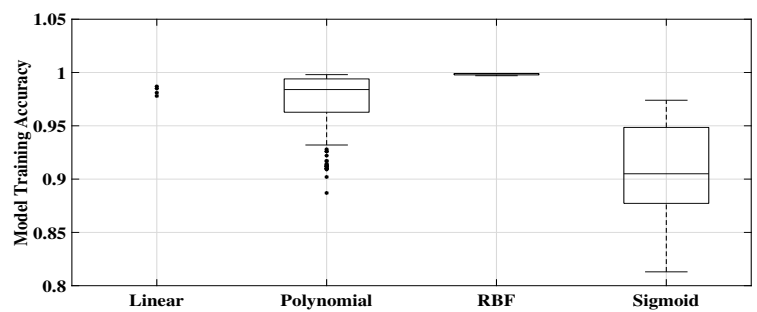

Fig. 19. Boxplot of Training Accuracy versus SVM Types.

processing requires instant processing, and communication devices must ensure higher data throughput. With the manually tuned testbed, our observed results indicate high precision in the training and test accuracies compared to the accuracies observed with multi sub-carrier based human motion detection systems. 


\section{CONCLUSION}

This study proposed a human motion recognition and identification system, implementing a single sub-carrier CW and SVM based pattern recognition system. The study employed a testbed for human motion data acquisition using state-of-the-art Software Defined Radios - NI-USRP 2901 and LabVIEW software. Operating at $5 \mathrm{GHz}$ frequency, a SISO channel created by transmitter (TX) and receiver (RX) enabled human motion patterns analysis using Support Vector Machine algorithm (SVM)to uncover human identities. Experiment was carried out in a controlled environment enabling us to set up environmental parameters as well as to fine tune the custom designed transmitter receiver. The findings in this study reveal that highly sensitive pilot RSS and SVM algorithm information can significantly help in recognizing human motion, which in turn guides to human identification (4 subjects) with the prediction accuracy of $98.92 \%$. Our proposed testbed system can be devised and validated on any of the commercially available NI-USRP added with LabVIEW Communication Design Software Suite.

The study anticipates a live motion recognition system in the future works, prototyping the current setup on an embedded device. SDRs in association with the high speed FPGA, support running SVM models directly. Therefore, this will serve as a guide to researchers interested in developing real time human motion recognition testbeds, thereby enabling them to test and evaluate system performances in other available ISM bands. Envisioned here is the development of more efficient, robust, and highly accurate human motion recognition systems, while using pilot signal RSS can also in human motion direction identification, still remains to be an open research problem.

\section{ACKNOWLEDGMENT}

This work was partially supported by the Kuwait Foundation for Advancement of Sciences (KFAS) under Grant PR1913NH-04. The paper adds a part of our previously published paper in the - 2020 IEEE Wireless and Networking Conference (WCNC).

\section{REFERENCES}

[1] N. Patwari and J. Wilson, "Rf sensor networks for device-free localization: Measurements, models, and algorithms," Proceedings of the IEEE, vol. 98, no. 11, pp. 1961-1973, 2010.

[2] K. Witrisal, P. Meissner, E. Leitinger, Y. Shen, C. Gustafson, F. Tufvesson, K. Haneda, D. Dardari, A. F. Molisch, A. Conti, and M. Z. Win, "High-accuracy localization for assisted living: 5g systems will turn multipath channels from foe to friend," IEEE Signal Processing Magazine, vol. 33, no. 2, pp. 59-70, 2016.

[3] M. Chen, K. Liu, J. Ma, X. Zeng, Z. Dong, G. Tong, and C. Liu, "Moloc: Unsupervised fingerprint roaming for device-free indoor localization in a mobile ship environment," IEEE Internet of Things Journal, pp. 1-1, 2020.

[4] _ "Moloc: Unsupervised fingerprint roaming for device-free indoor localization in a mobile ship environment," IEEE Internet of Things Journal, pp. 1-1, 2020.

[5] L. Chen, J. Hoey, C. D. Nugent, D. J. Cook, and Z. Yu, "Sensorbased activity recognition," IEEE Transactions on Systems, Man, and Cybernetics, Part C (Applications and Reviews), vol. 42, no. 6, pp. 790-808, 2012.

[6] F. Fereidoonian, F. Firouzi, and B. Farahani, "Human activity recognition: From sensors to applications," in 2020 International Conference on Omni-layer Intelligent Systems (COINS), 2020, pp. 1-8.

[7] X. Zhou, W. Liang, K. I. Wang, H. Wang, L. T. Yang, and Q. Jin, "Deeplearning-enhanced human activity recognition for internet of healthcare things," IEEE Internet of Things Journal, vol. 7, no. 7, pp. 6429-6438, 2020
[8] K. Xia, J. Huang, and H. Wang, "Lstm-cnn architecture for human activity recognition," IEEE Access, vol. 8, pp. 56855-56 866, 2020.

[9] M. Bennamoun, Y. Guo, F. Tombari, K. Youcef-Toumi, and K. Nishino, "Guest editors' introduction to the special issue on rgb-d vision: Methods and applications," IEEE Transactions on Pattern Analysis and Machine Intelligence, vol. 42, no. 10, pp. 2329-2332, 2020.

[10] R. Mondal, D. Mukherjee, P. K. Singh, V. Bhateja, and R. Sarkar, "A new framework for smartphone sensor based human activity recognition using graph neural network," IEEE Sensors Journal, pp. 1-1, 2020.

[11] J. Lu, X. Zheng, M. Sheng, J. Jin, and S. Yu, "Efficient human activity recognition using a single wearable sensor," IEEE Internet of Things Journal, pp. 1-1, 2020.

[12] O. Barut, L. Zhou, and Y. Luo, "Multi-task 1stm model for human activity recognition and intensity estimation using wearable sensor data," IEEE Internet of Things Journal, pp. 1-1, 2020.

[13] M. Zhou, Y. Wang, Z. Tian, Y. Lian, Y. Wang, and B. Wang, "Calibrated data simplification for energy-efficient location sensing in internet of things," IEEE Internet of Things Journal, vol. 6, no. 4, pp. 6125-6133, 2019.

[14] K. Woyach, D. Puccinelli, and M. Haenggi, "Sensorless sensing in wireless networks: Implementation and measurements," in 2006 4th International Symposium on Modeling and Optimization in Mobile, Ad Hoc and Wireless Networks, 2006, pp. 1-8.

[15] M. Youssef, M. Mah, and A. Agrawala, "Challenges: Device-free passive localization for wireless environments," in Proceedings of the 13th Annual ACM International Conference on Mobile Computing and Networking, ser. MobiCom '07. New York, NY, USA: Association for Computing Machinery, 2007, p. 222-229. [Online]. Available: https://doi.org/10.1145/1287853.1287880

[16] T. Chang, L. Wang, and F. Chang, "A solution to the ill-conditioned gps positioning problem in an urban environment," IEEE Transactions on Intelligent Transportation Systems, vol. 10, no. 1, pp. 135-145, 2009.

[17] B. Yang, L. Guo, R. Guo, M. Zhao, and T. Zhao, "A novel trilateration algorithm for rssi-based indoor localization," IEEE Sensors Journal, vol. 20, no. 14, pp. 8164-8172, 2020.

[18] P. Zhang, Z. Su, Z. Dong, and K. Pahlavan, "Complex motion detection based on channel state information and 1stm-rnn," in 2020 10th Annual Computing and Communication Workshop and Conference (CCWC), 2020, pp. 0756-0760.

[19] Z. Zhang, W. Nie, Y. Wang, and L. Xie, "Channel state information based indoor localization error bound leveraging pedestrian random motion," IEEE Access, vol. 8, pp. 153311-153321, 2020.

[20] M. T. Islam and S. Nirjon, "Wi-fringe: Leveraging text semantics in wifi csi-based device-free named gesture recognition," in 2020 16th International Conference on Distributed Computing in Sensor Systems (DCOSS), 2020, pp. 35-42.

[21] Z. Han, L. Guo, Z. Lu, X. Wen, and W. Zheng, "Deep adaptation networks based gesture recognition using commodity wifi," in 2020 IEEE Wireless Communications and Networking Conference (WCNC), 2020, pp. 1-7.

[22] Y. Chen, R. Ou, Z. Li, and K. Wu, "Wiface: Facial expression recognition using wi-fi signals," IEEE Transactions on Mobile Computing, pp. $1-1,2020$.

[23] W. Wang, A. X. Liu, M. Shahzad, K. Ling, and S. Lu, "Devicefree human activity recognition using commercial wifi devices," IEEE Journal on Selected Areas in Communications, vol. 35, no. 5, pp. 11181131, 2017.

[24] J. Yang, H. Zou, H. Jiang, and L. Xie, "Carefi: Sedentary behavior monitoring system via commodity wifi infrastructures," IEEE Transactions on Vehicular Technology, vol. 67, no. 8, pp. 7620-7629, 2018.

[25] Z. Fu, J. Xu, Z. Zhu, A. X. Liu, and X. Sun, "Writing in the air with wifi signals for virtual reality devices," IEEE Transactions on Mobile Computing, vol. 18, no. 2, pp. 473-484, 2019.

[26] H. Abdelnasser, K. A. Harras, and M. Youssef, "A ubiquitous wifi-based fine-grained gesture recognition system," IEEE Transactions on Mobile Computing, pp. 1-1, 2018.

[27] L. Zhang, Q. Gao, X. Ma, J. Wang, T. Yang, and H. Wang, "Defi: Robust training-free device-free wireless localization with wifi," IEEE Trans. on Vehicular Tech., vol. 67, no. 9, pp. 8822-8831, 2018.

[28] S. D. Domenico, M. D. Sanctis, E. Cianca, F. Giuliano, and G. Bianchi, "Exploring training options for rf sensing using csi," IEEE Communications Magazine, vol. 56, no. 5, pp. 116-123, 2018. 
[29] O. A. http://www.ni.com/en-lb/support/model.usrp 2901.html, "Ni usrp 2901."

[30] J. Mitsugi, Y. Kawakita, K. Egawa, and H. Ichikawa, "Perfectly synchronized streaming from multiple digitally modulated backscatter sensor tags," IEEE Journal of Radio Frequency Identification, vol. 3, no. 3, pp. 149-156, 2019.

[31] K. Wu, Jiang Xiao, Youwen Yi, Min Gao, and L. M. Ni, "Fila: Finegrained indoor localization," in 2012 Proceedings IEEE INFOCOM, 2012, pp. 2210-2218.

[32] S. A. Bhat, A. Mehbodniya, A. E. Alwakeel, J. Webber, and K. AlBegain, "Human motion patterns recognition based on rss and support vector machines," in 2020 IEEE Wireless Communications and Net- working Conference (WCNC), 2020, pp. 1-6.

[33] W. Buwei, C. Jianfeng, W. Bo, and F. Shuanglei, "A solar power prediction using support vector machines based on multi-source data fusion," in 2018 International Conference on Power System Technology (POWERCON). IEEE, 2018, pp. 4573-4577.

[34] N.-E. Ayat, M. Cheriet, and C. Y. Suen, "Empirical error based optimization of svm kernels: Application to digit image recognition," in Proceedings Eighth International Workshop on Frontiers in Handwriting Recognition. IEEE, 2002, pp. 292-297.

[35] Y.-D. Zhang and L. Wu, "Classification of fruits using computer vision and a multiclass support vector machine," Sensors (Basel, Switzerland), vol. 12, pp. 12 489-505, 122012. 